\title{
STEM Observation of eDNA as a Dominant Component of EPS in Pseudomonas aeruginosa Biofilm
}

\author{
Binbin Deng ${ }^{1}$, Subhadip Ghatak ${ }^{2}$, Subendu Sarkar ${ }^{2}$, Piya Ghatak ${ }^{2}$, David W. McComb ${ }^{1}$, Chandan K. \\ Sen $^{2}$ \\ 1. Center for Electron Microscopy and Analysis (CEMAS), College of Engineering, The Ohio State \\ University, Columbus, USA. \\ 2. Department of Surgery, The Ohio State University Wexner Medical Center, Columbus, USA.
}

Extracellular polymeric substances (EPS) are biofilm self-produced matrices that provide a protective shed for biofilm bacteria[1]. eDNA represents an important structural and functional component of EPS. Here we show that thread-like eDNA fill the space between bacteria throughout Pseudomonas aeruginosa prototypical strain PA01 biofilm (Figure 1).

Biofilm structures have been investigated by diverse microscopic methods, including fluorescence light microscopy, atomic force microscopy, transmission electron microscopy, and scanning electron microscopy [2-5]. Among them, confocal laser scanning microscopy is a powerful tool often used to study biofilm spatial structures. However, the resolution of confocal laser scanning microscopy limits its application to ultrastructural study of EPS. Most observation of eDNA and other EPS in biofilm are limited by resolution. In this work scanning transmission electron microscopy (STEM) is applied to the structural study of in vitro P. aeruginosa PA01 biofilm.

The biofilms were cultured on polycarbonate membrane in agar plate at $37^{\circ} \mathrm{C}$ for 48 hours. Biofilm discs were chemically fixed with $2.5 \%$ glutaraldehyde and $2 \%$ paraformaldehyde in $0.15 \mathrm{M}$ cacodylate buffer, and then en bloc stained with 2\% reduced osmium tetroxide. After dehydration and infiltration, samples were embedded in $100 \%$ durcupan resin and incubated at $65^{\circ} \mathrm{C}$ for 2 days. The resin embedded biofilm discs were processed to $90 \mathrm{~nm}$ ultra-fine sections by using a Reichert-Jung Ultracut E ultramicrotome (Leica, Wetzlar, Germany). The thin sections were picked up with a loop and put on 400 mesh copper grids. The grids were air dried and then coated with amorphous carbon $(\mathrm{t}=3 \mathrm{~nm})$ on both sides. Electron microscopy data were collected in STEM mode on a Tecnai F20 S/TEM (Thermo Fisher Scientific, Hillsboro) operating at $200 \mathrm{kV}$.

In $P$. aeruginosa PA01biofilm, eDNA was reported as the major component of EPS[6]. Our results showed that thread-like EPS presented throughout the PA01 biofilm, with especially large concentrations in the region close to the culture base (Figure 1). Recently, Turnbull et al. reported that explosive lysis of $P$. aeruginosa contributed significantly to the eDNA contents of biofilm [7]. Our images also indicated the thread-like structures had morphological similarity to the bacterial internal content (Figure 1), suggesting that the thread-like structures are DNA.

$P$. aeruginosa biofilm is frequently associated with chronic wound infection. Using STEM imaging techniques, we were able to gain an insight into the structures of $P$. aeruginosa PA01 biofilm. The structure provided the biofilm architecture for better understanding the biofilm EPS organization, which is likely to serve as structure basis for further therapeutic intervention [8]. 


\section{References:}

[1] JS Gunn et al, Journal of Biological Chemistry 291(24) (2016), p. 12538.

[2] V Berk et al, Science. 337(6091) (2012), p. 236.

[3] TA Smirnova et al, Microbiology 79(4) (2010), p. 413.

[4] K Drescher et al, Proceedings of the National Academy of Sciences 113(14) (2016), p. E2066.

[5] A Crespo et al, Frontiers in Microbiology 7(688) (2016).

[6] CB Whitchurch et al, Science 295(5559) (2002), p. 1487.

[7] L Turnbull et al, Nature communications 7 (2016).

[8] The authors acknowledge funding from National Institute of Health grants NR015676, NR013898, GM077185, GM108014, GM069589, DK076566, NS42617 and funding from Centre for Regenerative Medicine and Cell Based Therapies.



Figure 1. STEM image of PA01 biofilm showed thread-like eDNA structures. Scale bar $=2 \mu \mathrm{m}$. The EM sample section is $90 \mathrm{~nm}$ thick. The culture base is polycarbonate membrane. 